\title{
CCTS-based Business Information Modelling for Increasing Cross-Organizational Interoperability
}

\author{
C. Schroth ${ }^{1}$, G. Pemptroad ${ }^{2}$ and T. Janner ${ }^{1}$ \\ SAP Research CEC and the Institute for Media and Communications Management, \\ University of St. Gallen, Blumenbergplatz 9, 9000 St. Gallen, Switzerland \\ \{christoph.schroth, till.janner\}@\{sap.com/unisg.ch\} \\ 2 BOC Information Systems GmbH, Wipplingerstr. 1, 1010 Vienna, Austria \\ gregory.pemptroadaboc-eu.com
}

\begin{abstract}
The dissemination of e-Business solutions among small and medium sized enterprises (SMEs) is still very low due to various reasons. In the course of the GENESIS project, we aim at designing and deploying a novel e-Business platform that removes entry barriers for these SMEs. We present a living platform that grows over time and provides users with the possibility to seamlessly model and setup business relations with other users and execute these on the basis of a collaboratively designed standard. To achieve interoperability on both a business process and data level, we propose a novel, integrated modeling concept which is partly based on different existing standards such as the ISO 15000-5 CCTS specification (developed by UN/CEFACT). Our approach enables users to intuitively model and setup the unique processes and business documents they are willing to support while adhering to a common repository of data and process components. In this way, we ensurecross-organizational interoperability, facilitate reuse of existing components and leverage the emerging industry standard issued by UN/CEFACT.
\end{abstract}

\section{Introduction}

Although the adoption rate of e-Business technology in enterprises has significantly increased during the last decade, recent surveys indicate a limited penetration of such technology among small and medium-sized enterprises (SMEs) $[1,2]$. These SMEs - defined as companies that employ fewer than 250 persons and have an annual turnover not exceeding 50 million euro and/ or an annual balance sheet total not exceeding 43 million euro [3] - are facing hurdles that they are not able to overcome by the use of current e-Business technology. IT landscapes of typical SMEs consist of heterogeneous systems, reaching from Enterprise Resource Planning Systems (ERP) to spreadsheet-based island applications. Processes that are carried out between these SMEs typically require significant manual input due 
to media breaks, thereby preventing from seamless, cross-organizational eBusiness interoperability. This, in turn, results in unnecessarily high costs and missed business opportunities. These circumstances are the motivation to develop an e-Business platform in the course of the EU-funded project GENESIS [4] to allow typical SMEs to conduct their business transactions over the Web. A key factor towards the success of such a platform is the adequate handling of varieties in both business processes and documents according to individual requirements. At the same time, the platform has to be easily accessible for SMEs by hiding any technical complexity from them. The envisioned solution builds upon two major cornerstones:

First, a composite of standards issued by the UN/CEFACT [5], especially the ISO 15000-5 (formerly UN/CEFACT Core Component Technical Specification, CCTS) [6] form the technical basis of our e-Business platform. The effective support of the abovementioned technological foundation by means of an intuitive, flexible and business-oriented modelling method is the second major characteristic of our approach. In the course of the project, we designed a combined process and information modelling user interface that allows reuse of existing artefacts or components and enables their efficient customization according to current business needs.

The remainder of the paper is structured as follows. After presenting an overview of related research activities in Chapter 2 we proceed with a short overview of the EU-funded GENESIS project in Chapter 3. Chapter 4 will set the theoretical foundation in terms of providing a framework for business information modelling, which will to some extent be derived from literature and also from the requirements that arise from the experiences made during the GENESIS project. Chapter 5 presents in detail our integrated Business Information Modelling approach. A summary and outlook will be given in Chapter 6 .

\section{Related Work}

\subsection{Existing Projects}

During this work we refer to and have taken into account related work which references Business Information Modelling and which is originating from the following three areas:

Researchers from the University of Vienna have been involved in defining and extending methodologies that amend the upcoming UN/CEFACT e-Business framework. An Add-In for the UMM specification, for example, realizes a tool support for modelling of collaborative and cross-enterprise business processes and data based on the Unified Modelling Language (UML). In the area of CCTS, we refer to the work of Stuhec [7] who describes the Context-Driver-Principle for the efficient use and customization of CCTS-based business information.

Second, the European Integrated Project ATHENA [8] represents a related project dealing with the goal to establish a true collaboration between single businesses that are characterized by unique requirements both from the 
technological and from the business process perspective. The ATHENA project heads for contributing to interoperability by identifying and meeting a number of inter-related business, scientific \& technical, and strategic objectives. The development of new technology components, applications and services, but also the demonstration and testing of the results are in the focus of this project. ATHENA has been initiating an open, neutral and independent Enterprise Interoperability Centre (EIC) [9] to which all stakeholders, in both private and public sectors are invited to participate and to promote enterprise interoperability.

\subsection{Relevant Standards}

The work of the standardization bodies UN/CEFACT and OASIS is another major area our work is closely related to. To enhance business interoperability on a semantic level, we thoroughly investigated the ISO 15005-5 Core Component Technical Specification (CCTS) $[6,10]$ and numerous of closely affiliated standards issued by the UN/CEFACT. UBL [11] with its pre-defined business document description using Business Information Entities will be a further central part on which we refer to.

CCL \& CCTS. The Core Component Library (CCL) represents the repository for generic business data components, the so called Core Components. Based on the experiences gained in previous data standardization efforts, the CCL does not provide pre-determined, static or industry-specific data definitions, but comprises a huge set of context-agnostic, generally valid data templates (e.g. postal address, personal information) that are syntax-independent and represent the general business data entities which are commonly used in today's business processes. Major benefits of leveraging such a Core Component Repository include an increase of reuse of data elements during modelling and improved enterprise interoperability due to a common basis for business information description. UN/CEFACT envisions this library to grow and also change over time as users can either modify existing components or design and submit new Core Components in case the existing ones are not sufficient to fulfil the actual business requirements. The Core Component Technical Specification (CCTS) [6] is the associated method comprising meta-models and rules for the semantically unambiguous definition of business information on a syntax-independent level. This second building block of the UN/CEFACT stack provides users with guidelines for correctly naming and combining Core Components and introduces ways to apply context-specific restrictions on the generic data templates by adding semantic precision for a given business requirement. The generic data templates can thus be adapted and restricted to the individual requirements of users, thereby ensuring the scalability of the whole system.

An example for the use of syntax-free Core Components is as follows: a business Party is a so-called Aggregate Core Component (ACC) that consists of numerous components for representing data at leaf-level, such as "ID Number" etc. These basic data items that cannot be further decomposed into other components are called Basic Core Components (BCCs). The generic Party template comprises all BCCs that could ever occur in an arbitrary user context and is stored as an unstructured, syntax-free list. In case a Greek user desires to model a business 
document that comprises a Party entry, he is able to access the CCL, apply his Greek context and is provided an aggregation of data components that features only those BCCs that are relevant for his specific situation. This contextualized subset of the generic template is then called Business Information Entity (BIE). To semantically enrich a BIE even more, it is also possible to add qualifiers and, for example, transform a "ID number" into a "TaxPayer_ ID" to allow for differentiation from other "ID Number" instances. An address that is part of the aggregated information about a business Party is called ASCC since this data item can be decomposed into other, lower-level entities such as Street Names, Zip Codes etc.. In this way, all users have a common understanding of how the different parts of a business party are named and are yet able to use arbitrary syntactical representations for their business documents. Please refer to Figure 1 for a comprehensive description of the different CCTS based meta-model entities:

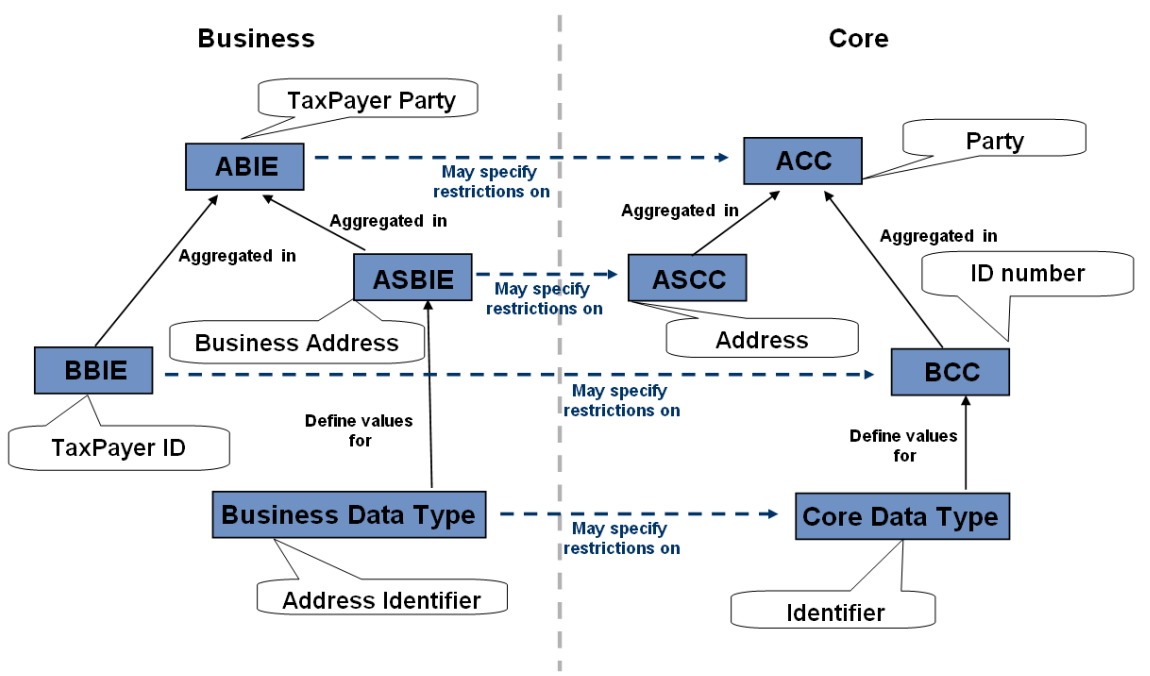

Fig. 1. CCTS Meta Model Entities

CDM, BMA, SBDH, CDT and Business Terms. The Context-Driver Methodology (CDM), the Business Message Assembly (BMA), Standard Business Document Header (SBDH), the Core Data Type (CDT) specification and the Business Terms are additional specifications that support the efficient and scalable use of the Core Components mentioned above and can be studied in detail in [10].

After elaborating on the CCTS meta-model, we now present the Universal Business Language (UBL) [11], which represents the second building block of our e-Business architecture: A royalty-free library of standard electronic XML business documents, UBL is designed to provide a universally comprehensible and well recognized commercial syntax for legally binding business documents. Developed by the Organisation for the Advancement of Structured Information Standards (OASIS), UBL's purpose is to provide: first, a library of XML schemas for reusable data components, such as Address, Item and Payment, which are the 
common data elements of everyday business documents. Second, a set of XML schemas for common business documents such as Order, Despatch Advice and Invoice that are constructed from the UBL library components and can be used in generic procurement and transportation contexts. Third, a set of processes and business rules associated with the business documents that define a context for their use.

UBL operates within a standard business framework such as ISO 15000-5 in order to provide a complete, standards-based infrastructure that can extend the benefits of existing EDI systems to businesses of all sizes. As the first standard implementation of UN/CEFACT CCTS, the UBL Library is based on a conceptual model of numerous information components known as Business Information Entities (the collection of which we refer to as UBL Common Library). These components are assembled into specific document models such as Order and Invoice (UBL business documents), which are then transformed in accordance with UBL Naming and Design Rules into W3C XSD schema syntax.

\section{The GENESIS project}

\subsection{Project Overview}

The EU-funded GENESIS project [4] aims at increasing the adoption of eBusiness specifically among SMEs. As the central composite to be designed and also implemented in the course of this project, an interoperable platform is developed that enables seamless and cross-organizational collaboration among business partners. We examine and model the processes and information entities exchanged by the SMEs with other enterprises (B2B), governmental institutions (B2G) or Intermediaries (B2I, eg. Banks). For detailed information on the major cornerstones of the GENESIS project we refer to [4].

\subsection{Architectural Overview}

Figure 2 depicts the overall system architecture. A central server provides the necessary functionality to connect the users to the system, to manage the communication between them and to provide an environment for business processes and document modelling and execution. Apart from that, it follows a store-and-forward approach that takes into account that SME users are not necessarily continuously connected to the Internet. The server is able to temporarily store business messages and forward them to other users as soon as they are connected.

Following the paradigm of a Service-Oriented Architecture (SOA) [12] in combination of the essentials of the ebXML approach [13], the required functionality is provided as a set of Web Services. The server also contains a registry and repository part (containing the GENESIS Common Library (CL)), which is devoted to storing templates for the supported processes and business 
documents of all registered SMEs and supporting the negotiation of business conditions among SMEs.

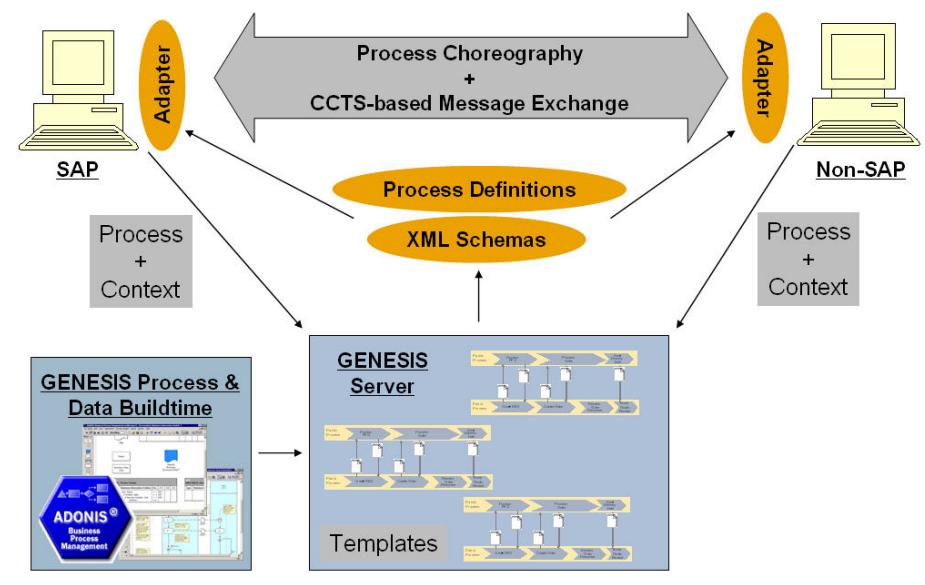

Fig. 2. GENESIS Overall architecture

When connecting to the system, clients are supposed to send their respective user context (formalized according to the existing guidelines of the CCTS) and the names of supported processes to the server. The server then passes back process definitions and XML schemas (representing the business documents) to the client that are appropriate for the individual context. Apart from that, clients are enabled to connect to the system in two different ways. A fat client, called adapter, will be implemented on the side of the users to connect legacy IT systems to the GENESIS system. This adapter realizes a machine-to-machine interface for an efficient automated transaction processing. In case users do not wish to leverage existing IT systems, a Web-based thin client will be provided as a machine-to-human interface to access the system. During design time, a new environment (GENESIS Process \& Data Buildtime) supports users with modelling business information in a way that facilitates cross-organizational interoperability and re-use of existing information entities. The following sections concentrate on this modelling environment and thoroughly describe underlying technologies as well as use cases.

\subsection{Basis of the GENESIS Modelling Method}

The first work packages of the GENESIS project highly involve SMEs to analyse their requirements related to business documents exchanged within different kind of business processes (B2B, B2G, or B2I). We decided to use graphical models for capturing this information because they allow optimal representation of process flows or document assemblies. It is important to mention that Business Information Modelling should encompass not only the description of the document content (information entities and their relationships) but also the context in which the document is used (activities, actors, rules). 
As a large effort had to be devoted to modelling activities, it was important to ensure efficient modelling activities. This can mainly be achieved using a wellsuited modelling method. We define below the four major components of a modelling method: A modelling framework indicates what is being modelled, providing the required modelling scope depending on the project goals. Description levels of the Enterprise Architecture, architectural views and granularity of framework elements have to be specified. A modelling technique defines how the framework elements have to be modelled. This defines a description language, a corresponding graphical notation, and a meta-model definition that contains modelling rules (e.g. how modelling constructs can be linked together). It can also be a combination of several techniques, each of them used for a specific part of the framework. The result is a set of modelling constructs (stereotypes) with attributes (tagged values) having a graphical representation (shape and visualized values), which are made available in one or more model types (diagrams). Model-related mechanisms are a set of tools that allow modellers to manipulate models and their content. This includes modelling rules checker, quality-assurance functions, productivity-enhancing features, simulation algorithms, transformation scripts, publishing solutions, or import/export mechanisms (e.g. XSD Schema Generation). A modelling approach (procedure, practice) can be both an input and output of the three previous elements. It specifies the procedure or practice for the modelling activities within a project, defining also roles and responsibilities. When does a modelling activity take place? Who is in charge of which modelling activity? Which models and constructs are being used for that modelling activity?

The development of the GENESIS modelling method combined both state-ofthe-art and empirical studies. A critical success factor for the novel modelling method is taking into account the modellers' heterogeneous modelling and analysis skills. This led us to the development of a project-specific modelling method that should enable the modellers to quickly become familiar with the modelling technique and to easily capture project-relevant information. Modellers' feedback during test phases was the best way to guarantee a user-friendly modelling technique. Existing approaches were identified and analyzed; some of them were tested and integrated if they could provide a proper modelling solution and a real value for the project. In the following sections, we will further describe the modelling method chosen for the project.

\section{The GENESIS Integrated Approach}

In this paper, we propose novel techniques and approaches that significantly improve crosss-organizational interoperability. The establishment of a common repository of information modeling building blocks and an intuitive modeling approach that guides modelers along an unambiguous methodological path are the crucial factors facilitating business information interoperability. The following sections will highlight the cornerstones of our modelling method and will also explain its collaborative and evolutionary characteristic. 


\subsection{Business Information Modelling Technique}

According to the modelling framework we retained for the project, a business document is the key element between processes, rules and information entities; the document is used by actors within activities of a business process and business/legal rules may impact the use or the content of a document, composed of business information entities. We managed to develop a modelling technique for the GENESIS project that offers an integrated modelling of all these elements.

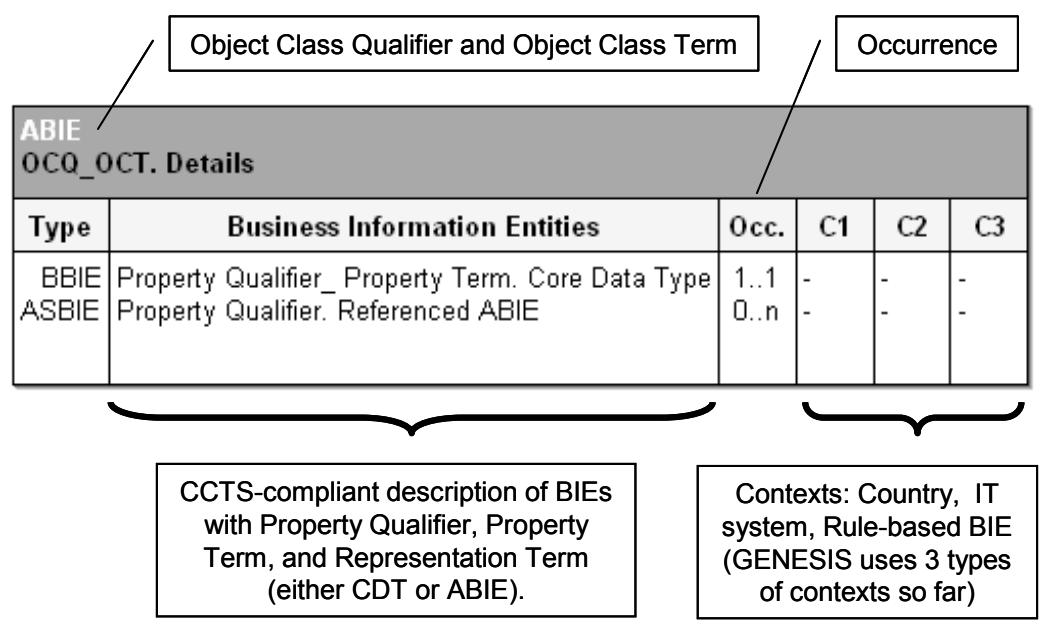

Fig. 3. “ABIE” Modelling Construct in ADONIS ${ }^{\circledR}$

Two existing notations (ADONIS BPMS 3.81 and BPMN 1.0) that already offer intuitive features for linking processes and documents have been leveraged as a foundation of our modelling technique. However, both notations do not provide CCTS-compliant decomposition of business documents into information entities, so that we needed to develop appropriate extensions. For the representation of CCTS compliant documents, UML Class Diagrams have turned out to be an inadequate choice: we identified two major weaknesses of the UML notation: the documentation of contexts is not possible and the graphical representation of ABIEs is not user-friendly. Thus, we developed a specific technique, which is rather close to CCTS, and provide a new model type called "Business Information Model", which offers both intuitive and comprehensive description of business documents.

We propose the use of mainly three modelling artefacts: The Specific Business Document (SBD) summarizes information about a complete and user-specific document and is referenced in user-specific process models. The Generic Business Document (GBD) can be considered as a consolidated version of several userspecific documents and features all data items that occur in any of the affiliated SBDs. The idea behind the establishment of GBDs was to create data templates that can be used by all clients and only need to be restricted according to user context to exactly match the respective users' business requirements. GBDs are then referenced from harmonized collaboration process model. Finally, Business 
Information Entities (BIEs) are those components that are used to assemble both SBDs and GBDs. As shown in Figure 1, BBIEs, ABIEs and ASBIEs are used by the CCTS meta-model. Figure 3 visualizes the graphical representation of an ABIE: the Business Information Entities, their Occurrence and information about their usage in different contexts can be specified in an intuitive manner. The documentation of BIEs is supported by using an alternative, spreadsheet-like feature to enter all CCTS-compliant information (see Fig. 4). Information such as "Alternative business terms" or "Definition" are documented, but not displayed in this summarized graphical view. In compliance with the CCTS, the Representation Term of a BBIE has to be chosen among a pre-defined list of Core Data Types. For linking existing ABIEs, the modeller has to reference an ABIE from the UBL Common Library in order to specify an ASBIE.

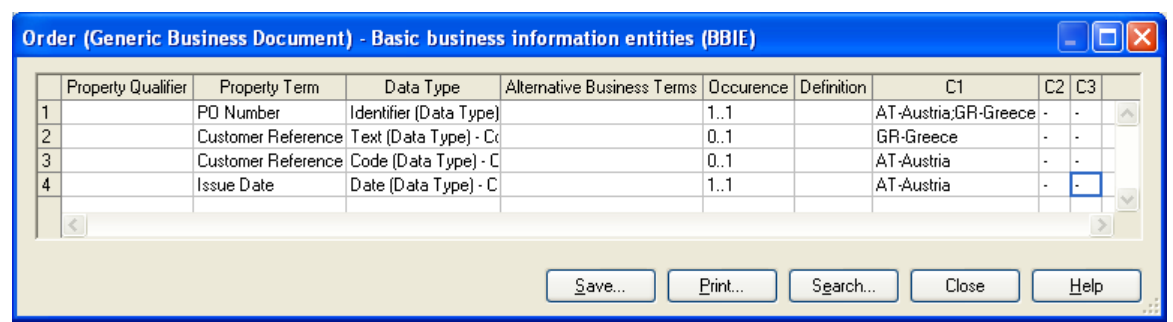

Fig. 4. Extract of the BBIE Description Feature in $\mathrm{ADONIS}^{\circledR}$

\subsection{Integrated Modelling Approach}

The modelling approach we selected for this project identified three roles involved in describing documents:

Business Users who daily deal with business documents can identify the documents, the context of use and provide a basic description of information entities. At this stage, we only expect to get the name of a BIE, to know if it is mandatory and to understand simple formatting rules if exist. CCTS concepts are not used so far. The corresponding document could also be attached to the model in order to share this example with other modellers. Business users are also supposed to provide some metrics about the utilization of the documents (e.g. size, processing time). A second group of users, the Business Analysts, further detail documents by using the CCTS compliant artefacts (BBIEs, ABIEs, ASBIEs, and CDTs). They should be skilled in Business Information Modelling according to the CCTS approach. During the adapter development phase, IT Specialists would refine CDTs into Business Data Types (BDTs) by applying restrictions according to the CCTS concepts. Restrictions usually depend on country-specific rules or ITsystem-specific configurations. The documentation of restrictions ensures the development of adapters for a proper exchange of data between diverse IT systems and the GENESIS platform.

One of the challenges we wanted to respond to was to have one single modelling environment for all three types of modellers. In this way, there is one unique entry point for gathering information about a business document rather than 
having separated descriptions in different tools or different files formats, which would lead to redundancies or inconsistencies. Therefore, we developed a collaborative solution on the basis of ADONIS $^{\circledR}$, which can be seen as an Integrated Modelling Environment [14] that has been customized according to the GENESIS modelling method. It offers a repository of business document descriptions and can generate XSD schemas for each document.

\subsection{Design Phase}

After elaborating on both the modelling technique and the approach of our novel modelling method we now present ways to design and setup a first basis of commonly comprehensible documents that adhere to the CCTS meta-model and are based on the UBL Common Library and its affiliated business documents. We refer to this basis of business documents that are designed by users as the GENESIS Common Library. The documents in scope of the first design phase are the ones used within a selection of frequently used collaboration processes stemming from three categories: B2B (e.g. Ordering), B2G (e.g. VAT Statement) and B2I (e.g. Statement of Account). After the selection of processes to be modelled for the project, a group of Business Users mapped these processes to identify the business documents and attachments - structured or not - that are exchanged during such a business process. The context of use of a document was thus clearly described.

Then, Business Analysts modelled relevant structured business documents using BIEs. The integrated modelling environment contained a set of reference models with the UBL Business Documents (UBL BD) and the UBL Common Library (UBL CL), which were very important during the design phase of B2Brelated documents. Even if several countries and languages are represented through the business users involved in the project, the language that has been selected for modelling is English. This is the first reason why business analysts referred to the UBL reference models: they found reference terms in order to name documents and information entities with usual and correct English terms. UBL was also used for identifying the document structures and information entities. Having reference models definitely facilitated the analysis of business users' documents (SBD) by comparing and mapping them with UBL documents. The CCTS-fields "Alternative Business Terms" and "Description" are particularly important to understand what a BIE stands for. A copy of the UBL CL was attributed to each Business User, renamed into USER Common Library (USER CL) that could be adapted to show user-specific description of BIEs. The Business Analysts had two options when working with BIEs from the UBL CL: to delete a BIE that is not relevant for the SBD or to refine a BIE by modifying some CCTS-fields like "Data Type" or "Occurrence". If a BIE of the SBD could not be described by a BIE of the UBL $\mathrm{CL}$, Business Analysts were describing the SBD with a new BBIE or a new ASBIE (in this case, inserting a new ABIE in the USER CL).

It finally allowed us to make a gap analysis between users' documents and UBL documents, and to move from the UBL CL to a GENESIS CL, after consolidation of all users' Common Libraries. This step has been done by the GENESIS team and consisted in consolidating several SBDs into a single Generic 
Business Document (GBD) as a super-set of SBDs (see Fig. 5). In complement to the GENESIS CL with reusable ABIEs, we also get a list of GENESIS Business Documents (GENESIS BD), described according to the CCTS concepts.
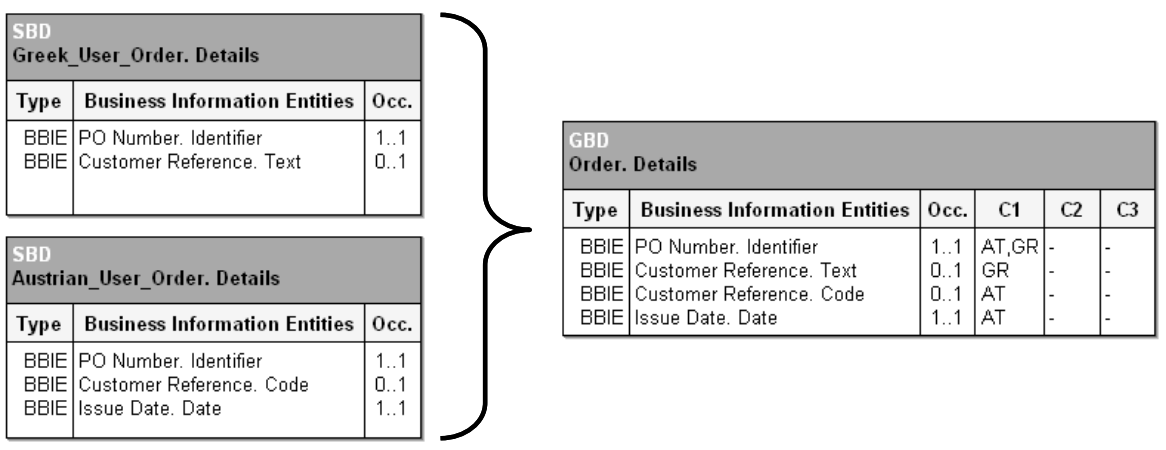

Fig. 5. Example of Consolidation from SBDs to GBD ( $\mathrm{C} 1=$ Country $)$

\subsection{Operational Phase}

After presenting an exemplary use case of our modelling method, we now show the functionality during the operational phase of the GENESIS platform. First of all, our modelling environment features an XML generator that maps the mere semantic data models into UBL compliant XSD files. The Web Services that build the technical foundation of the GENESIS platform then reference these files and thus integrate them into the collaboration business processes established between trading partners. During the execution of processes, the modelling environment becomes inactive and is only used in case of change requests triggered by a user.

The repository of semantic building blocks (GENESIS CL) for information modelling presented above is operated in a collaborative and evolutionary way. In case new users connect to the system, they might have different business requirements and thus desire to publish and use new components. The UN/CEFACT has issued a technical specification [15] that describes a way for building a registry and repository that allows users to upload new data modelling artifacts and make them publicly available. A UN/CEFACT-compliant registry also defines interfaces for stakeholders that are responsible for the maintenance of existing artefacts (including harmonization and identification of redundancies).

\section{Conclusion}

In this work, we described our approach to improve enterprise application interoperability on the basis of the upcoming UN/CEFACT e-Business framework. We concentrated on evaluating the advantages of using a collaborative and extensible repository of Business Documents and their Information Entities as a basis for modelling shared business references. With the help of a use case 
scenario, we highlighted the benefits of this approach during both design and operational phases. Future publications will elaborate on the integration of the proposed business information modelling method and the modelling of collaborative business processes. The resulting comprehensive design environment that is currently being developed within the GENESIS project will have great potential to increase cross-organizational interoperability, to reduce modelling complexity and thus lowers the e-Business entry barriers for SMEs.

\section{References}

[1] W. L. Lomerson, L. C. McGrath, and P. H. Schwager: An examination of the benefits of e-business to small and medium size businesses, in Proceedings of the 7th Annual Conference of the Southern Association for Information Systems, 2004.

[2] European Commission: The European e-Business Report 2005 edition - A portrait of e-business in 10 sectors of the EU economy, http://ec.europa.eu/enterprise/, 2006

[3] European Commission: The new SME definition - user guide and model declaration, http://ec.europa.eu/enterprise/, 2006

[4] GENESIS project, http://www.genesis-ist.eu, 2006

[5] The United Nations Centre for Trade Facilitation and Electronic Business, www.unece.org/cefact, 2006

[6] UN/CEFACT: Core Component Technical Specification V2.01, http://www.untmg.org, 2006

[7] G. Stuhec: How to solve the business standards dilemma - CCTS key model concepts, SAP Developer Network (SDN), http://sdn.sap.com, 2006

[8] ATHENA: Athena Project, http://www.athena-ip.org/, October 2006

[9] EIC Community: Enterprise Interoperability Centre. http://www.eic-community.org/, 2006

[10] T. Janner, A. Schmidt, C. Schroth, and G. Stuhec: From EDI to UN/CEFACT: An evolutionary path towards a next generation e-business framework, Proceedings of The 5th International Conference on e-Business 2006 (NCEB2006), 2006

[11] OASIS: Universal Business Language (UBL), http://www.oasis-open.org/, 2006

[12] G. Alonso, F. Casati, H. Kuno and V. Machiraju: Web Services Concepts, Architectures and Applications. Springer Verlag, 2004

[13] A. Kotok, D. R.R. Webber. ebXML: The new global standard for doing business over the web. New Riders, 2001

[14] H. Kühn; F.Bayer; S.Junginger; D.Karagiannis: Enterprise Model Integration, Proceedings of the 4th International Conference EC-Web 2003, Dexa 2003, Prague, Czech Republic, September 2003, LNCS 2738, Springer Verlag, pp. 379-392

[15] I. Bedini and F. Bourge: UN-CEFACT - Registry Specifications V1.0 Draft, http://www.untmg.org, 2006 